\title{
Fremd unter Kollegen: Wissenschaftskooperation mit Osteuropa
}

von Ralf Rytlewski

Als ein Beitrag zur Reform der Bildungssysteme der osteuropäischen Länder gründeten private Stiftungen 1998 die Bonner Initiative „Johann Gottfried Herder" mit dem Ziel, deutsche Dozenten im Ruhestand an ausländische Hochschulen zu entsenden. Zunächst als Hilfsmaßnahme gedacht, verbindet sich das Programm inzwischen mit einer aktiven deutschen Außenwissenschaftspolitik. Über Möglichkeiten, Schranken und Wirkungen einer mehrjährigen Gastdozentur der Stiftungsinitiative in Rumänien wird im doppelten Kontext einer gemeinsamen Entwicklung der Hochschulsysteme und des Trends zu einer forcierten Internationalisierung der Wissenschaft berichtet.

As a contribution to the reform of the higher education systems in Central and Eastern European countries, a number of private foundations joined forces to establish the Bonnbased "Stiftungsinitiative Johann Gottfried Herder", which aims at enabling retired German academics to teach at universities and colleges in the region. While this was initially conceived as an effort to support ongoing reform efforts, it nowadays also represents a valuable part of the German external science and research agenda. This report sheds light on the options, obstacles, and effects of these multi-annual visiting professorships, looking specifically at experiences from Romania and outlining two concurrent developments: the intertwined development of European higher education systems and the trend towards an accelerated internationalisation of academic research.

\section{Die Ausgangssituation}

\section{Deutschland in europäischer Mittellage}

Die Deutschen sahen sich am Ende der Ost-West-Spaltung anders als ihre europäischen Nachbarn vor eine doppelte Aufgabe gestellt. So ging es zum einen darum, binnenstaatlich aus den unterschiedlichen Erfahrungen und Prägungen von Ost wie West eine gemeinsame Lebens- und Arbeitsform zu entwickeln, während zum anderen die Republik gefordert war, zur Mittellage zwischen den Westeuropäern und den slawischen Völkern und Nationen Europas zurückzufinden. Wenngleich viele Deutsche ein distanziertes Verhältnis zur eigenen Nation aufwiesen und vielfach nicht Nationen, sondern Regionen als künftige Grundeinheiten Europas empfahlen, betrachteten die Nachbarn das vereinte Deutsch- 
land als den deutschen Nationalstaat, ganz unabhängig davon, ob die Deutschen „es wollen oder nicht und obwohl sie es nicht mehr gewohnt sind“. ${ }^{1}$ An ihn richteten sich sofort vielfältige ökonomische und kulturelle Erwartungen, vor allem Offenheit im gesamteuropäischen Rahmen. Ohne großzügige, dem MarshallPlan vergleichbare Hilfe - so der zu jener Zeit noch tschechoslowakische Präsident Václav Havel 1991 anlässlich der Verleihung des Karls-Preises in Aachen „werden wir zwar nicht auskommen“, doch der Annäherung Mittel- und Osteuropas an den Westen läge das Bekenntnis zur Kultur und zu den Werten Europas zugrunde, „die wir als die unseren empfinden, weil wir lange Jahrhunderte an ihrer Schaffung beteiligt waren“.2 Zweifellos findet Deutschland seitdem schon aufgrund seiner zentralen Lage und seiner Geschichte die besondere Aufmerksamkeit seiner Nachbarn und übernahm - gemessen an der Vergangenheit mehr politische Verantwortung in Europa und den größeren Teil der Hilfen im West-Ost-Transfer. Auf die doppelte Aufgabe antwortete die deutsche Seite mit der Einheit von Vereinigungs- und pro-Europa-Politik unter dem geglückten Leitbild eines „europäischen Deutschlands“. Auf etwa die Hälfte aller finanziellen Leistungen des Westens an die neuen unabhängigen Staaten schätzte Außenminister Klaus Kinkel 1992 den deutschen Beitrag. So engagierten sich die Deutschen im Rahmen der Transformation Mittel-, Ost- und Südosteuropas sowie der GUS-Staaten von Anbeginn, zumal sie ,am eigenen Leibe [erfahren], was es heißt, aus dem Stand nach 40 Jahren Kommandowirtschaft pluralistische, marktwirtschaftliche Strukturen aufzubauen". ${ }^{3}$ Aus deutscher Perspektive waren und sind die mittel- und osteuropäischen Staaten einer häufig genutzten Formel zufolge nach Jahrzehnten der Trennung auf dem Weg „zurück nach Europa“, während in diesen Ländern selbst die Ansicht überwog, Europa auch zu Zeiten der Blockkonfrontation nie verlassen zu haben.

Der Transfer war vielfältig und reichte von kurzfristiger Versorgung mit Lebensmitteln, Energie und Medikamenten über private Spenden und Kredite bis hin zu den Kompensationszahlungen beim Abzug der Westtruppen des Warschauer Pakts. Das Bundesministerium der Finanzen bezifferte den entsprechenden Aufwand im Zeitraum von 1989 bis 1993 auf insgesamt 125 Mrd. DM. $^{4}$

1 Henrich, D.: Nach dem Ende der Teilung. Über Identitäten und Intellektuelle in Deutschland, Frankfurt a.M. $1993,7$.

2 Rede zur Karlspreisverleihung am 9.5.1991 in Aachen, in: Auswärtiges Amt (Hg.): Deutsche Außenpolitik nach der Einheit 1990-1993. Eine Dokumentation, Meckenheim 1994, 43-45, 45.

3 Rede am 25.5.1992 in Berlin, in: Deutsche Außenpolitik, a.a.O.,161-164, 163.

4 Bundesministerium der Finanzen, in: Deutsche Außenpolitik, a.a.O., 453-457, 457. 
Anhand des nachfolgend im Zentrum stehenden Beispiels Rumäniens und den 1993 gültigen mittelfristigen Vorhaben wurden als materielle Schwerpunkte deutlich:

- die Vermarktung landwirtschaftlicher Produkte, Partnerschaften im Handwerk, Hilfen bei der Wirtschaftsreform,

- der Auf- und Ausbau medizinischer Einrichtungen,

- Maßnahmen der gesellschaftspolitischen Bildung und im Rahmen der sich verändernden Sozialstruktur sowie

- die Schaffung eines Studien- und Fachkräftefonds. ${ }^{5}$

Im Mittelpunkt aller Aufmerksamkeit standen als Oberziele der deutschen Aktivitäten die Förderung der Transformation hin zur Marktwirtschaft sowie die Unterstützung des demokratischen und gesellschaftlichen Wandels. Kulturelle und wissenschaftliche Themen kamen nur indirekt zur Sprache, etwa dort, wo es um professionelle Beratung und Training ging. Daraus ist allerdings nicht auf Versäumnisse zu schließen. Im Falle der DDR reagierten Universitäten und Kultureinrichtungen auf die Öffnung der deutsch-deutschen Grenze geradezu springflutartig mit Reisen und Kontakten, um im fachkollegialen Gespräch Informationen über die jeweilige Arbeitssituationen auszutauschen, woraus sich wiederum einzelne Kooperationsprojekte ergaben bzw. bereits in den 1980er Jahren geknüpfte Verbindungen gefestigt und fortgeschrieben werden konnten. Kaum eine Universität in Deutschland verweigerte sich entsprechenden Kontakten. Nach einem ersten Verebben dieser „Primärhilfen“ entwickelten dann die wissenschaftlichen Großorganisationen, wie der Deutscher Akademischer Austauschdienst (DAAD) und Deutsche Forschungsgemeinschaft (DFG), im Frühjahr 1990 spezifische Förderprogramme, die den Hochschulen und Akademie-Instituten der DDR helfen sollten, ,Defizite aufzuholen“ und einen „Beitrag zum Reformprozess zu leisten“. ${ }^{6}$ Diese Fördermöglichkeiten wurden später auf die Länder Mittel-, Ost- und Südosteuropas ausgeweitet. Zunächst ging es jedoch darum, Gastdozenten und Sachmittel in die DDR zu entsenden. Eine verbesserte Ausstattung der Institute in Ostdeutschland sollte dortige Forscher ,ermutigen, in ihrem Land weiterzuarbeiten, um einem weiteren Abwandern vor allem von Nachwuchswissenschaftlern vorzubeugen“" 7

5 Bundesministerium für wirtschaftliche Zusammenarbeit: Laufende Vorhaben in Mittel-, Ost- und Südosteuropa, Kaukasus und Mittelasien, in: Deutsche Außenpolitik a.a.O., 458-460,459.

6 DAAD-Pressenotiz 5/90, 1.

7 forschung - Mitteilungen der DFG 1/90, 28. 


\section{Grenzüberschreitende Wissenschaftskooperation}

Die damit angesprochenen wissenschaftsinternen und -externen Zusammenhänge erwiesen sich als äußerst komplex. Wenn Wissenschaft einerseits ihrem europäischen Ursprung folgend über allgemein akzeptierte Merkmale wie Rationalität, Methodik und Archivierung geradezu regelhaft an Internationalität gewinnt, wird sie andererseits doch durch den Wettbewerb von Staaten und Gesellschaften ähnlich regelhaft attrahiert und beeinflusst. Wissenschaft und ihre Wirkungsstätten sind Teil der Landeskultur und zugleich der internationalen Gemeinschaft der Wissenschaftler.

Analog lassen sich Wissenschaftskooperationen von zwei grundverschiedenen Positionen aus betreiben. Der erste Standpunkt hebt die Internationalität der Wissenschaft hervor, die grenzüberschreitende Zusammenarbeit verlangt. Die zweite Position geht vom nationalen Forschungspotenzial als einem entscheidenden Faktor beim Wettbewerb zwischen den Ländern (und etwaigen Auseinandersetzungen) aus, sodass es gilt, die wirtschaftliche, militärische und gesellschaftliche Präsenz zu steigern. Wie stets sind Mischformen möglich und üblich, wenn etwa in einem Themenfeld nationale Forschungsvorhaben unterschiedlicher Länder auf internationaler Ebene erörtert und abgestimmt werden. Heute fehlt es nicht an Stimmen, die, wie insbesondere die deutsche Hochschulrektorenkonferenz (HRK), schon den weiteren Übergang von der internationalen zur transnationalen Gemeinschaft der Weltuniversitäten konzipieren und propagieren, einer Gemeinschaft von Universitäten, die sich, ,in ihrer Gesamtheit als Akteur(e) in einem globalen Kontext" ${ }^{68}$ verstehen.

Derart konträre Ziele stellen jedes konkrete grenzübergreifende Projekt unter einen Spannungsbogen, unterhalb dessen Zusammenarbeit stets unterschiedlich interpretierbar wird. So mag die häufig am Beginn einer Kooperation stehende Literaturhilfe demjenigen, der die Lehrbuchsammlung übergibt, die international gültigen Paradigmen des Faches repräsentieren, während sie für den Empfänger das nationale Curriculum von außen infrage- oder vor der Studierendenschaft sogar bloßzustellen vermag. Irritationen schon auf dieser Ebene können sich noch zuspitzen, wenn darüber hinaus Gastdozenten nicht thematische Ergänzungen lehren, sondern die Kernbereiche eines Curriculums übernehmen. Stolpersteine drohen dabei insbesondere in den Geistes- und Sozialwissenschaften, da

8 Lenzen, D.: Die deutschen Hochschulen in der Welt und für die Welt - die Internationale Strategie der HRK, in: Hochschulrektorenkonferenz: Bausteine für den internationalen Erfolg, Bonn 2012, 16-20, 17. 
den darin versammelten Fächern im Rahmen der Ideenkonkurrenz zwischen Ost und West eine entscheidende Rolle zukam.

Im deutschen Fall gingen die Meinungen in dieser Frage auseinander. Für den bayerischen Kultusminister Hans Zehetmair, dessen Amt sich im Übrigen ebenso wie die bayerischen Universitäten höchst intensiv für Ost-West-Begegnungen einsetzte, war es zweifelsfrei, dass aufgrund einseitiger marxistisch-leninistischer Orientierung ,die Voraussetzungen eines internationalen Austausches in diesen Fächern derzeit nicht gegeben“" sind. ${ }^{9}$ Stimmen vor allem aus den Hochschulen wollten weniger pauschal vorgehen. Tatsächlich kam es in den ersten Monaten der „Neugierde und Expansionslust“"10 nicht nur in Berlin zu vielfältigen Austauschbeziehungen und Kontakten, und dies sogar in der unter systemischen Gesichtspunkten besonders exponierten Politikwissenschaft. Dieses Fach hatte sich in der Sowjetunion und ihren Bündnisstaaten neben dem Lehrgebäude des Wissenschaftlichen Kommunismus ansatzweise etablieren können, die entsprechenden Lehrstühle waren nach der Grenzöffnung für beide Seiten willkommene Anknüpfungspunkte. In Ostberlin und den neuen Bundesländern erwies sich die Lage als noch komplizierter, da die SED eine Abspaltung und Parallelisierung des Faches nicht zugelassen hatte. Die deutsche und internationale Ausprägung der Politikwissenschaft konnte in der DDR nur insgeheim rezipiert werden. ${ }^{11}$

Die zahlreichen Einzelinitiativen von unten, also auf Ebene der Universitäten und Forschungsinstitute, waren in Deutschland, mittelfristig gesehen, von geringer Wirkung, was auf eine Reihe sehr unterschiedlicher Gründe zurückgeführt werden kann. Vor allem der im deutsch-deutschen Einigungsvertrag von 1990 mit Art. 38 vollzogene Strategiewechsel, den Prozess der ,,notwendigen Erneuerung von Wissenschaft und Forschung" in thematischer, sachlicher und nicht zuletzt personeller Hinsicht mit einer Evaluierung aller DDR-Einrichtungen durch Fachgutachter des bundesrepublikanischen Wissenschaftsrats zu beginnen und in etwas mehr als Jahresfrist auch schon zu beenden, erwies sich als bedeut-

9 Zehetmaier, H.: Das Zusammenwachsen der deutschen Hochschulen im europäischen Rahmen - politische Aspekte, in: Eisenmann, P./Schmirber, G. (Hg.): Deutsche Hochschulen und Europa, Regensburg 1990, 136-144, 138.

10 Riedmüller, B.: Wissenschaftsstadt Berlin. Hochschulen und Forschung im Vereinigungsprozeß, in: Sü $\beta$, W./Rytlewski, R. (Hg.): Berlin. Die Hauptstadt, Berlin 1999, 715-738, 716.

11 Dies allerdings intensiv, wie der Vortrag des Leipziger Marxisten Rendgen nahelegt : Die Politikwissenschaft im Erneuerungsprozeß der ehemaligen DDR, Ms. (11.1.1991). 
sam. ${ }^{12}$ Diese Weichenstellung zu einer Steuerung von oben erleichterte es über alle örtlichen Initiativen hinweg, westliche Wissenschaftsmodelle der Erneuerung zu Grunde zu legen.

Von der deutsch-deutschen Entwicklung ist jene in den anderen Ländern Mittelund Osteuropas zu unterscheiden. Nicht der radikale und schnelle Modellwechsel war dort das Ziel, sondern die Öffnung von Forschung und Lehre (samt ihrer institutionellen Voraussetzungen), wodurch Räume für verschiedene wissenschaftspolitische Orientierungen entstanden. Es bot sich einerseits Raum für Anpassungen an westliche Standards, zugleich aber auch für ein Beharren auf Zügen des sowjetisch-russischen Modells und schließlich auch für Experimente gemäß der jeweiligen nationalkulturellen Ausgangssituation. Hier öffnete sich ein weites thematisches Feld für Kontakte mit westlichen Wissenschaftlern und deren Interessen am Transformationsprozess. Wie die Hochschulrektorenkonferenz (HRK) für 1993 mitteilte, fanden sich zu diesem Zeitpunkt bereits 866 Kooperationsbeziehungen deutscher Hochschulen mit Partnereinrichtungen in Mittel-, Ost- und Südosteuropa, überstrahlt von den deutsch-polnischen Beziehungen, innerhalb derer Wissenschaftler aus 80 polnischen und 124 deutschen Einrichtungen zusammenfanden. Nun ist nicht jede Quantität von gleichem Gewicht und erweist es sich bekanntlich oft als schwierig, von Quantität auf Qualität zu schließen. Doch dokumentiert die nachfolgende Übersicht (Tab. 1), welch große Bedeutung der direkten Nachbarschaft nicht nur im Bereich der Außenpolitik und im gesellschaftlichen Leben, sondern auch im internationalen Wissenschaftsbetrieb zukam (und bis heute zukommt).

Es zahlte sich aus, dass deutsche Universitäten auch in der Zeit der Blockgegensätze Wissenschaftskontakte hatten pflegen können - häufig initiiert und getragen von den Instituten der Osteuropa-Forschung. So vermochte es die Freie Universität im Westen Berlins, dank eines engagierten universitären Außenamts, Kontakte über die Grenzen des Kalten Krieges hinweg mit Wissenschaftlern auf der sowjetischen Seite zu etablieren - und dies trotz der wissenschaftspolitischen Linie der Sowjetunion, für West-Berlin abweichend von den Vier-MächteVereinbarungen einen Sonderstatus als eigenes staatliches Gebilde durchzusetzen. An dieser Frage hatte sie 1961 das zweite deutsch-sowjetische Kulturabkommen ,platzen“ lassen. Literaturaustausch, Kongressteilnahmen und der we-

12 Vertrag zwischen der Bundesrepublik Deutschland und der Deutschen Demokratischen Republik über die Herstellung der Einheit Deutschlands - Einigungsvertrag 1990, in: Bulletin Nr. 104, 6.9.1990, 877888, Art. 38. 
chselseitig organisierte Besuch einzelner Wissenschaftler standen lange Zeit im Mittelpunkt. Spätestens in den 1980er Jahren wurde es darüber hinaus üblich, jüngere Mitarbeiter für mehrere Monate bis zu einem Jahr für Studienaufenthalte zu entsenden, so seitens der Staatsuniversität Leningrad an die Freie Universität Berlin.

Tabelle 1: Kooperationsbeziehungen deutscher Hochschulen mit Hochschulen und Wissenschaftseinrichtungen in Mittel- und Osteuropa im Jahr 1993 (Anzahl der Kooperationen und Beteiligungen)

\begin{tabular}{l|c|c|c}
\hline Land & Kooperationen & Deutschland & $\begin{array}{c}\text { Länder Mittel- } \\
\text { und Osteuropas }\end{array}$ \\
\hline Bulgarien & 59 & 38 & 22 \\
\hline Estland & 34 & 26 & 8 \\
\hline Kroatien & 15 & 13 & 3 \\
\hline Lettland & 31 & 25 & 7 \\
\hline Litauen & 31 & 25 & 7 \\
\hline Polen & 322 & 124 & 80 \\
\hline Rumänien & 37 & 28 & 18 \\
\hline Slowakei & 55 & 45 & 3 \\
\hline Slowenien & 16 & 15 & 29 \\
\hline Tschechische Republik & 121 & 72 & 50 \\
\hline Ungarn & 157 & 90 & 14 \\
\hline
\end{tabular}

Quelle: Hochschulrektorenkonferenz/Smolarczyk, R./Referat C4 (Hg.): Mittel- und Osteuropa, Zentralasien, 2012.

Der langjährige wissenschaftliche Sekretär der Deutschen Forschungsgemeinschaft, Wolfgang Kasack, beschrieb das Grundmuster aller dieser Beziehungen treffend: Jeder Wissenschaftler reise nicht nur aus wissenschaftlichen Gründen, er wolle vielmehr auch Land und Leute selbst sehen und hören. Er ist „Nehmender und Gebender zugleich. Nehmender beim Kennenlernen der Institute, der laufenden Forschungen, Gebender in Einzelvorträgen oder Gastvorlesungen, in jeder Diskussion“. 13

13 Kasack, W.: Formen und Probleme der wissenschaftlichen Beziehungen, in: Geyer, D.: Wissenschaft in kommunistischen Ländern, Tübingen 1967, 278-298, 282 


\section{Idealisten mit Erfahrung}

\section{Stiftungsinitiative Johann Gottfried Herder}

Idealisten mit Erfahrung, so wurden anlässlich der dritten „Bilanztagung“ der Stiftungsinitiative 2008 in Bonn jene Wissenschaftler bezeichnet, die als deutsche Gastdozenten in Transformationsländern wirkten. Wissenschaftler im Ruhestand zu Lehraufträgen für ein oder zwei Semester an fremde Universitäten zu vermitteln und zu fördern, diese wissenschaftspolitische Idee verfolgten der DAAD und Wissenschaftsministerien einzelner Bundesländer bereits $1990 \mathrm{im}$ Rahmen eines Programms, das darauf zielte, die an den DDR-Universitäten betriebenen Wirtschafts-, Rechts- und Sozialwissenschaften besser in die Lage zu versetzen, sich aktiv an der Umgestaltung von Wirtschaft und Gesellschaft zu beteiligen. Jahre später rückten private Förderstiftungen diese Idee in das Zentrum einer Initiative, Finanzmittel in einer 1998 gegründeten Stiftungsinitiative unter dem insbesondere in Osteuropa wohl bekannten Namen Johann Gottfried Herder zusammenzutragen, um mit Hilfe dieses Fonds Emeriti und Pensionäre in Transformationsländer zu entsenden. Den Wunsch nach intensiveren Kontakten hatten osteuropäische Kollegen 1997 auf dem Hochschulverbandstag in Dresden geäußert. Die nachfolgende Initiative übernahmen ab 1999 in einer ersten Phase die Alfried Krupp von Bohlen und Halbach-Stiftung, die Fritz Thyssen Stiftung, die Gemeinnützige Hertie-Stiftung, die Robert Bosch Stiftung GmbH, der Stifterverband für die Deutsche Wissenschaft und die ZEIT-Stiftung Ebeling und Gerd Bucerius. Die Stiftungssumme von 5 Mio. DM sollte in den nächsten drei Jahren 150 bis 180 Gastdozenturen ermöglichen. Von den Gastdozenten erwarteten die Initiatoren ,wichtige Impulse bei der Qualifizierung und Ergänzung von Forschung und Lehre sowie der Curriculumsentwicklung“. ${ }^{14}$ Sie setzten sich das Ziel, zur Reform des Studiums und der Lehre in den Ländern Mittel- und Osteuropas und der Gemeinschaft Unabhängiger Staaten (GUS) beizutragen und waren sich bewusst, auch auf unbekanntes Terrain zu geraten. „Aber wer sollte risikobereit sein, wenn nicht private Stiftungen und Persönlichkeiten, die sich nicht mehr wegen eines ,Karrierevorteils' verdingen“, war sich Heinz-Rudi Spiegel vom Stifterverband sicher. ${ }^{15}$

14 Rogall, J.: Programm, in: Stiftungsinitiative Johann Gottfried Herder (Hg.): Begegnungen im Osten Europas, Bonn 2004, 8-12, 8f.

15 Passgenaue Vermittlung. Interview mit dems., in: Begegnungen a.a.O., 108-109, 109. 
Die Stiftungen wählten als Trägerorganisationen den DAAD und die HRK, wodurch die Initiative die Form einer privat-öffentlichen Partnerschaft annahm. Da private Stiftungen in der Regel nur Anschubfinanzierungen ermöglichen, ist ihre Rolle befristet, was zu Wechseln unter den Stiftern führte und inzwischen die öffentliche Hand, über das Auswärtige Amt, die gesamte Finanzierung übernehmen ließ. War das Programm in der zweiten Phase (2003 bis 2009) darauf konzentriert, südosteuropäische Hochschulen mit Blick auf die EU-Mitgliedschaft und den europaweiten Übergang zu Studienabschlüssen des Bachelor (BA) und des Master (MA) zu unterstützen, so erfolgte 2009 die weltweite Öffnung des Programms. Materiell konnten in den ersten drei Jahren 188 Semesterdozenturen in 14 Ländern gefördert werden, bis zum Sommersemester 2004 waren es bereits 313 Dozenturen in 17 Ländern. ${ }^{16}$ Mit Blick auf die vertretenen Fächer lagen zunächst die Blöcke der Sprach- und Kulturwissenschaften einerseits und die der Mathematik, der Natur- und Ingenieurwissenschaften sowie der Medizin andererseits gleich stark vorn, die Rechts-, Wirtschafts- und Sozialwissenschaften rückten erst später vor. Die Evaluierung des Südosteuropaprogramms ergab, so Stefan Hormuth, Präsident des DAAD, dass die Gastdozenturen insbesondere für „die Aufrechterhaltung der deutschsprachigen Studienprogramme (...) eine überragende Rolle gespielt haben“. ${ }^{17}$

Jede Gastdozentur beruht auf einer Übereinkunft zwischen dem Dozenten, der aufnehmenden Hochschule, deren Mitglied er/sie während der Dozentur mit den daraus folgenden landesüblichen Ansprüchen wird, und der Stiftungsinitiative. Die Gasthochschule hat ihr Interesse auch mit einem Beitrag zu den Kosten der Dozentur zu bekunden. Grundsätzlich kann, wie schon 1990, von jeder Seite der Anstoß zu einer Dozentur erfolgen, konkrete Vermittlungsprozesse wurden lagespezifisch mehrfach verändert.

\section{Fremdsein als Wechselwirkung}

Soziologisch gesehen bewegt sich der Gastdozent der Herder-Stiftungsinitiative (im Folgenden Herder-Dozent genannt) als ein Fremder an seiner neuen Wirkungsstätte. Er ist mit diesem Ort weder verwandtschaftlich noch lokal verbunden und tritt, da nicht befangen in lokalen Interessenlagen und Parteiungen, in der Haltung des Objektiven auf, der ungebunden und unüblich frei auf der uni- 
versitären Bühne erscheint. Auf Georg Simmel (1908) geht zurück, den Fremden somit in der Einheit von Innerhalb und Außerhalb zu sehen. ${ }^{18}$ Als Mitglied der grenzüberschreitenden Wissenschaftlergemeinschaft bewegt der Gastdozent sich im „Innerhalb“, als Deutscher in Osteuropa zugleich im „Außerhalb“. Er verkörpert die Nahtstelle, an der beides wirkt; Fremdsein ist eine ,,positive Beziehung, eine besondere Wechselwirkungsform“. ${ }^{19}$

Als Herder-Gastdozent zog der Autor samt seiner mitlehrenden Ehefrau im Herbst 2006 an den Lehrstuhl für Politische Wissenschaft der West-Universität in Temeswar (Timisoara) und die dort gebildete Deutsche Abteilung Internationale Beziehungen und Europa-Studien (DAIBES). Der Historiker und Kenner der deutsch-rumänischen Beziehungen Vasile Docea initiierte 2005 den gleichnamigen deutschsprachigen Studiengang, den er seitdem leitet. Die 1944 noch von König Mihai I. aus der Linie Hohenzollern-Sigmaringen per Dekret gegründete und 1948 eröffnete Universität in Temeswar liegt im Westen Rumäniens, im Banat, und trägt folglich den Namen West-Universität. Es handelt sich dabei um eine Volluniversität mit rd. 20000 Studierenden und elf künstlerischen, geistes-, sozial- und naturwissenschaftlichen Fakultäten, darunter die Fakultät der Politikwissenschaften, Philosophie und Kommunikationswissenschaften, aus deren Lehrstühlen eine Hochschulreform jüngst Abteilungen bildete. Auch die Germanistik ist seit 1956 an der West-Universität beheimatet, unter Einschluss eines Zeitraums, in dem dort aus den beiden deutschen Staaten entsandte Lektoren zeitgleich lehrten. Die West-Universität, die benachbarte Technische Universität und weitere staatliche und private Hochschulen geben Temeswar das Flair einer Universitätsstadt.

Das Bildungsniveau der rumänischen Bevölkerung differiert wie überall zwischen Stadt und Land, ist im europäischen Vergleich aber insgesamt und insbesondere im mittleren Segment recht hoch: Es wird für 27 Prozent der 25- bis 64jährigen als niedrig eingeschätzt, für 62 Prozent als mittel und für elf Prozent als hoch. ${ }^{20}$ Nach 1989 nahm Rumänien an der ungewöhnlich starken osteuropäi-

18 "Der Fremde ist ein Element der Gruppe selbst, nicht anders als die Armen und die mannigfachen „,inneren Feinde“ - ein Element, dessen immanente und Gliedstellung zugleich ein Außerhalb und Gegenüber einschliesst.“, ders.: Der Fremde, in: Landmann, M. (Hg.): Das individuelle Gesetz, Frankfurt a.M. $1968,63-70,63 \mathrm{f}$.

19 Ebd., 63.

20 Europäische Kommission: Wachsende Regionen, wachsendes Europa. Vierter Bericht über den wirtschaftlichen und sozialen Zusammenhang, Luxemburg 2007, 191. Zum Vergleich die portugiesischen und die deutschen Bevölkerungsanteile : niedrig 73\% bzw. 17\%, mittel 14\% bzw. 59\%, hoch $13 \%$ bzw. $25 \%$, ebd. 191, 179; vgl. auch Reisz, R.D./Stock, M.: Zwischen Expansion und Kontraktion. Zur 
schen Hochschulexpansion teil, die den Abbau von Studienplätzen während der 1980er Jahren korrigierte. Zwar bleiben die Studienplätze weiterhin zentral quotiert und sind dafür prinzipiell gebührenfrei, doch ist es den Hochschulen inzwischen gestattet, gegen Gebühren zusätzliche Plätze anzubieten. Die Universitäten partizipieren an Förderprogrammen der Europäischen Union (EU) sowie am European Credit Transfer System (ECTS), das schon 1996/97 beschlossen wurde. Entsprechend dieser Ausgangslage ging es für den Herder-Dozenten 2006 nicht mehr darum, institutionell Grundlegendes zu schaffen, sondern ein bereits gegebenes und ministeriell genehmigtes Curriculum unter Einsatz der in Deutschland üblichen politik- und sozialwissenschaftlichen Standards sowie der eigenen langjährigen Erfahrung in Lehre und Forschung umzusetzen, wobei die Akzente auf der Schärfung des Lehrprogramms und seiner didaktisch anspruchsvollen Anwendung lagen. Im Ergebnis gelangten der Autor und seine Frau als Fremde in eine sozial- und geisteswissenschaftliche Gemeinschaft, die sich aus den Studierenden, der Kollegenschaft und den mit der Universität verbundenen Teilen der Stadtgesellschaft zusammensetzte. Auf allen drei Ebenen machten wir während acht Semestern spezifische Erfahrungen, die im Zentrum der folgenden Darstellung stehen.

\section{Lehren und Lernen}

Für Studierende im ersten und zweiten Studienjahr boten wir Einführungen in die Politikwissenschaft und in die Politische Ökonomie sowie Seminare, unserem Verständnis nach Proseminare, zu den Theorien und Strategien der Europäischen Integration sowie zur Menschenrechtsthematik an. Im Laufe der Jahre traten zahlreiche weitere Lehrgebiete hinzu, darunter methodische Grundlagen, wissenschaftliche Arbeitsweisen und - materielle - Themenbereiche wie die Politische Anthropologie, die Ethik und Soziologie internationaler Beziehungen, ideengeschichtliche Grundlagen oder auch Theorien des Nationalismus - Lehraufgaben, die nur vor dem Hintergrund breiter Lehrerfahrungen zu leisten sind.

Der Jahrgang 2006/7 war mit 73 Studierenden in der deutschsprachigen Abteilung besonders stark besetzt, von ihnen entrichteten über die Hälfte Gebühren. Der nachfolgende Studienjahrgang setzte sich dann aus 33 Studierenden zusammen, danach pendelte sich die Zahl bei etwa 25 Studierenden ein. Da in Jahrgängen studiert wird, ähneln sich die Studierenden in vielem, was im günstigen Fall 
eine vergleichsweise intensive Lernsituation befördert. Ihre Kenntnisse in der Fremd- oder Muttersprache erwerben die jungen Menschen auf deutschsprachigen Schulen, die die Sprache auch nach der Abwanderung der meisten Rumänien-Deutschen während und nach der kommunistischen Diktatur anboten, jetzt primär für Schüler und Schülerinnen mit rumänischer Muttersprache. Besondere Bedeutung und Aufmerksamkeit erlangen die Spezial-Abteilungen der deutschen Lyzeen. Sie führen zur Deutschen Allgemeinen Hochschulreife, die in Deutschland den Universitätszugang ohne weitere Sprachprüfungen garantiert. ${ }^{21}$ Von 32 Abiturienten des Abschluss-Jahrgangs 2006/7 des Nikolaus-Lenau-Lyzeums in Temeswar wählten zehn ein Auslandsstudium, alle anderen rumänische Programme, davon wiederum zehn den örtlichen deutschsprachigen Studiengang Internationale Beziehungen und Europa-Studien. ${ }^{22}$ Dieser und ähnliche Studiengänge treffen auf eine starke Nachfrage, da das geschrumpfte deutsche Schulnetz seit Jahren nun vor allem nicht-deutschen Schülern eine erfolgreiche Studienund im Weiteren auch Berufsperspektive offeriert.

Was erwarten Studierende vom Fremden aus Deutschland? Zumindest sollte er zuverlässig, qualitätsbewusst und anspruchsvoll auch sich selbst gegenüber auftreten, natürlich pünktlich und im Übrigen fähig sein, aus einer Schraube einen Motor zu bauen. ${ }^{23}$ Diese allgemeinen Erwartungen der Rumänen an die Deutschen schützen im konkreten Fall allerdings nicht vor dem Zusammenprall zweier Lehr- und Lernweisen, die der Dozent in der Mitte zwischen Innerhalb und Außerhalb nicht ohne weiteres ausgleichen kann. Will die heimische Seite vor allem umfangreiches repetierbares Wissen vermitteln und hat damit durchaus auch Erfolg (sodass das Vorlesungspublikum, nennt man nur nebenbei den Namen Joseph A. Schumpeters, unisono mit dem Begriff schöpferische Zerstörung reagiert), so konzentriert sich die fremde Seite, der der Gastdozent von Herkunft verpflichtet ist, zwar ebenfalls auf Wissensvermittlung, wohl aber in Problemund Lösungszusammenhängen.

Diese nicht nur didaktischen Pole sind anfangs Ausgang nicht weniger arbeitstechnischer Differenzen und Missverständnisse. So darf eine rumänische Lehr-

21 Die Lyzeen in Timisoara, Brasov, Sibiu und anderswo sowie die Deutsche Schule in Bukarest sind Zentren deutscher Bildung und Kultur. Im Schuljahr 1990/91 umfasste das deutsche Schulnetz 165 Kindergärten, 139 Grund- und Gymnasialschulen und 15 Lyzeen, vgl. Kleininger, N.: Deutsche Schulen nehmen Brückenfunktion wahr, in: VDA (Hg.): Rumäniendeutsche zwischen Bleiben und Gehen, 3.Aufl., Sankt Augustin 1991, 20-21, 20.

22 Nikolaus Lenau Lyzeum Temeswar (Hg.): Jahrbuch 2006/2007, Temeswar 2007, 54f.

23 Vergleichsweise gehörte es zum Credo des rumänischen Mannes, seinem Auto so anverwandt zu sein, dass er es jederzeit und jedenorts ohne Fachhilfe es zu reparieren in der Lage sei. 
veranstaltung durchaus lauter sein, während die deutsche Seite die Ruhe gemeinsamen Überlegens herbeizuführen sucht. Nicht alle Beteiligten sind bereit, dem Wechsel in der verfolgten Didaktik zu folgen, bis hin zu einer,,inneren Trennlinie“ in der Studentenschaft des Jahrgangs. Einer der Gründe dafür mag am Rekrutierungssystem liegen, das die allermeisten Abiturienten direkt in ein Studium wechseln lässt, da die Alternative einer Berufsausbildung noch immer weitgehend unbekannt ist. Zwanzig bis fünfundzwanzig Prozent des hier nachverfolgten Jahrgangs verhielten sich zunächst desorientiert, desinteressiert und gelegentlich durchaus auch destruktiv. Manche von ihnen fanden erst gegen Studienende zu einer konstruktiveren Haltung. Jenseits dieser Besonderheit fand sich im Übrigen viel Einzelwissen, Engagement, Fleiß und bei nicht wenigen Studierenden auch eine andauernde Begeisterung, die wiederum die Lehre stimulierte.

Als ein Erbe der kommunistisch-russischen Ausbildungsweise hat sich das Memorieren von diktierten, abzuschreibenden oder neuerlich elektronisch übermittelten Texten bis in die Gegenwart erhalten, auch wenn Lehrpläne inzwischen eine Projektorientierung und vernetztes Denken fordern. Dennoch gilt bis heute: Reine Textwiedergaben erleichtern dem Dozenten die mechanische Textüberprüfung und befördern die Bewertung mit einheitlichen Maximalnoten für alle. Lernende und Lehrende können aus der Memorierungsmethode leicht Nutzen ziehen. Vor diesem Hintergrund wird verständlich, wenn Studierende im Plagiat kein Arbeitsproblem erkennen wollten. Unter denen, die unmotiviert auf der anderen Seite der Trennlinie verharrten, sollen diejenigen, so geht die Fama, die in der Nacht vor dem Abgabetermin einer Hausarbeit diese in der allerkürzesten Zeit, etwa zwei Stunden, aus dem Internet zusammenkopierten, die geheimen Gruppenhelden gewesen sein. Wenn bis zur Hälfte der Studierenden so oder ähnlich vorgeht, beschreibt das ein schwer wiegendes strukturelles Defizit der üblichen Arbeitsmethodik. Unsere Antwort konnte nur sein, in alle Veranstaltungen das Thema der wissenschaftlichen Arbeitsmethoden einzufügen, was positiven Widerhall auch bei den Kollegen und den Nachwuchskräften hervorrief. Durch unsere Veranstaltungen mussten die Studierenden, für viele überraschend, lernen, auf zwei Wissenschaftsklavieren zu spielen, was manchen ein Schock, anderen eine Chance war.

Der vom Gastdozenten praktizierte dialogische Lehrstil nahm in den folgenden Semestern zeitweise den Charakter von sozialwissenschaftlichen Werkstätten an. Höhepunkte waren dabei Gastvorlesungen, deren Erörterungen vor- und nachbereitet, auch benotet wurden. Auf diese Weise kamen die Studierenden mit einem halben Dutzend rumänischer und deutscher Parlamentarier ins Gespräch. Ein 
anderer Weg, die Studierenden stärker mit der gesellschaftlichen Umwelt in Verbindung zu bringen, waren extern durchgeführte Seminare, in denen vor Ort praktische Aufgaben im Temeswarer Rathaus, im deutschen Konsulat, in Räumen des Demokratischen Forums der Deutschen im Banat, im privat getragenen Revolutionsmuseum der Stadt und nicht zuletzt im Deutschen Kulturzentrum gestellt wurden, woraus sich neben allen Einsichten fachliche Kontakte und Praktikaplätze ergaben. $^{24}$

Da die Resonanz erfreulich stark war und eine Vorlesungsstunde für studentische Gespräche mit Gästen kaum Zeit ließ, entwickelten wir, basierend auf der finanziellen Unterstützung durch die Stiftungsinitiative, den Typ der außeruniversitären Fachkonferenz, die Studierende über ein Wochenende auf dem Land zusammenführte. Die erste dieser Konferenzen widmete sich 2007 in Georgiu Baie dem zivilgesellschaftlichen Aufbruch Rumäniens. Die benotete „Großwerkstatt“ wurde zum „Meilenstein“ insofern, als die Studierenden in ihr nach eigenem Urteil erstmals mit methodisch betriebener Politikwissenschaft konfrontiert wurden und an wie in ihr lernten. Die Praxis und der Nutzen analytischen Vorgehens standen danach nicht mehr in Frage.

Zur Steigerung des Leistungsniveaus trug auch bei, dass ausführliche Sprechstunden aufgrund universitärer Raumnöte in der Privatwohnung offeriert wurden. Hier wurden die Studierenden zudem mit jenen Lehrbüchern konfrontiert, die bald nach Ankunft des Gastdozenten über einem Rundbrief von der deutschen Kollegenschaft erbeten wurden. Da deutschsprachige Lehrbücher anfangs gänzlich fehlten, finanzierten darüber hinaus deutsche Sponsoren den Einkauf weiterer Bände und zahlreicher Lehrmaterialien. Diese Form von Internationalität der Wissenschaft beeindruckte die Studentenschaft, die sich dadurch ernst genommen fühlte. Aus dieser Praxis entwickelte sich ein jour fixe am Freitagnachmittag, der informell auch der Erörterung ,moralischer“ Fragen, wie denen nach historischer Schuld, Geschlechtergerechtigkeit oder demokratischer Tugend, galt; angesichts der tiefen und keineswegs überwundenen sozialmoralischen

24 Zum Beispiel waren im Rathaus die zuständige Referentin, das örtliche Büro der GIZ, der Berliner Stadtforscher Prell und ein Service-Unternehmer daran beteiligt, die Aufgabe der Studierenden, das Profil Temeswars einer breiteren Öffentlichkeit zugänglich zu machen, zu erörtern und anschließend zu begleiten; dem war ein Seminar zu Trends der Stadtentwicklung vorausgegangen. In ähnlichem Zuschnitt wurden weitere Politikfelder wie Geschlechterpolitik, Erinnerungspolitik oder Sozialpolitik bearbeitet. 
Krise, in die die Diktatur des Ehepaars Ceaucescu des Land gestürzt hatte, konnte dies nicht erstaunen. ${ }^{25}$

Bei insgesamt aufsteigender Linie stand im Hintergrund stets auch das Problem der Notengerechtigkeit. Sie erwies (und erweist) sich als ein spezifisches Dilemma schon im deutschsprachigen Schulunterricht, spätestens im letzten Schuljahr. Wer an eine deutschsprachige Universität gehen will, ist daran interessiert, eine gute Abiturnote zu erreichen, um sich Chancen im Numerus-ClaususAuswahlverfahren zu eröffnen, während diejenigen, die ein rumänisches Studium beginnen wollen, vorausgreifendes Fachwissen erwerben müssen, da in den Universitäten meistens fachgebundene Zugangsprüfungen $\mathrm{zu}$ bestehen sind und die Abiturnote nur von geringem Einfluss ist. Diese Differenz erweitert sich, wenn die Noten eher in traditioneller rumänischer Methode gehandhabt werden, während sie in den deutschsprachigen Studiengängen und Lehrveranstaltungen als Leistungsnachweise im vollen Notenspektrum von eins bis zehn vergeben werden und dabei häufig unterhalb des Notenschnitts geraten, was sich für die Studierenden nachteilig bei der Vergabe von Leistungsstipendien oder an Leistung gebundenem Wohnraum auswirken kann. Semester für Semester entwickeln sich Unterschiede zwischen Noten, die als geschönt, manipuliert, gekauft angeprangert werden, und solchen, die individuelle Anstrengungen bestätigen, zu einen universitären und auch öffentlichen Thema. ${ }^{26}$ Erreicht dieses dann die offizielle Bildungspolitik des Landes, wird gern darauf verwiesen, dass zum Notenmissbrauch stets zwei Seiten, eine gebende und eine nehmende, gehören.

\section{Multikulturell behaust}

Rumänien gerät in den Blick der deutschen Öffentlichkeit häufig nur über die emotional hoch aufgeladenen Bilder vom blutsaugenden Grafen Dracula, zeitgenössisch variiert am Beispiel Ceaucescu, von darbenden Waisenkindern in ärmlichen Heimen und von dürren, müden Pferden, die schäbige Fuhrwerke über unbefestigte Dorfstraßen ziehen. Jedes dieser Bilder spricht von einer deutlichen Ahnungslosigkeit, die der jahrzehntelang erkennbaren wechselseitigen Informationsabschottung geschuldet sein mag, dem Land und der Gesellschaft Rumä-

25 Dazu aufschlussreich die sozio-politische Studie Mungiu, A.: Die Rumänen nach '89, hg. v. Friedrich Ebert Stiftung, Bukarest 1996; literarisch gefasst u.a. durch H. Müller und R. Wagner.

26 Im Sommersemester 2007 begann auf der Plattform des Studiengangs ein anonymer Vers: „Rosen sind rot,/Veilchen sind blau,/gibt es Gerechtigkeit an unserer Uni?/Man weiß es nicht genau./Von höherer Bildung/nichts zu sehen,/solange manche Dubioses begehen,/den Anspruch dezimieren,/das Niveau reduzieren/... unter aller Sau.“ 
niens aber nicht gerecht wird. Auch ist der weit verbreiteten Annahme entgegenzuwirken, mit der Auswanderung der Deutschstämmigen sei deren Wirken in der rumänischen Wirtschaft, Gesellschaft und Politik beendet. Die Nachwirkungen der Ausgewanderten sind vielmehr erheblich und mehren den Einfluss der etwa 60.000 deutschstämmigen Bewohner, die im Lande blieben und sich ökonomisch in Landwirtschaft und Mittelstand, kulturell in Häusern der Bildung, der darbietenden Künste und der Medien sowie politisch im Demokratischen Forum der Deutschen als relevante Minderheit zeigen. Der Herder-Dozent der Temeswarer Universität gerät ohnehin in eine Stadt, deren Straßen und Bauten vom Vorbild Wiens geprägt sind, das auch für die Namen der Vorstädte Josefstadt und Elisabethstadt einsteht. Im Zentrum erkennt man noch die ehemalige Festung, die erstmals 1171 erwähnt wird, in der Folge ungarischer, osmanischer und österreichischer Herrschaft diente und erst 1920 an Rumänien fiel. Heute ist es eine Stadt mit multikulturellem und polyreligiösem Flair in einer ökonomisch höchst erfolgreichen Region. Ist die Multilingualität der Marktfrauen schon einnehmend, so nicht minder die des katholischen Bischofs im barocken Dom oder die des protestantischen Pastors in der ungarischen Kirche nebenan, die die Gottesdienste zweisprachig, wenn nicht gar dreisprachig zelebrieren.

In einer derartigen Stadtgesellschaft hat der Fremde es leicht, sich als akzeptiert zu empfinden. So wird zu einem Zeichen der Behausung, wenn beim allwöchentlichen Konzert der „Filarmonica Banatul“ nicht nur Studenten beginnen, sehr freundlich zu grüßen, sondern auch Honoratioren der Stadt. Die deutsche community ist hier stets präsent, etwa über den Konsul, den Präsidenten des deutschen Wirtschaftsclubs, Geschäftsleute, Kollegen, Ärzte, Musikstudenten oder Journalisten. Eine wichtige Rolle spielt auch das zweisprachige Deutsche Staatstheater, das unter dem Dach des vom Wiener Atelier Fellner und Helmer entworfenen Rumänischen Nationaltheaters exzellente Inszenierungen bietet, ergänzt um das Gymnasium, den Wirtschaftsclub, die historischen und kulturellen Veranstaltungen im Adam Müller-Guttenbrunn-Haus des Demokratischen Forums der Deutschen, deren 1992 gegründeter Jugendtrachten-Verein „Banater Rosmarein“ sich Gustav Mahlers bewegenden Ausspruch: „Tradition ist nicht Anbetung der Asche, sondern Weitergabe des Feuers“" zum Motto setzte. Das Rathaus unter dem deutschsprachigen Bürgermeister Ciuhandu pflegt zudem den Austausch mit zahlreichen deutschen Orten und vor allem der Partnerstadt Karlsruhe.

Wie ein Spiegel begleitet die in Osteuropa einzig verbliebene deutschsprachige Tageszeitung, die „Allgemeine Deutsche Zeitung“ (ADZ), das Schicksal der deutschen Minderheit. Trotz Enteignung und Deportationen blieben 1948 rd. 
350.000 Deutschstämmige im Land. Die Zeitung wurde 1949 unter dem Titel „Neuer Weg“ gegründet und galt für viele einmal als beste Tageszeitung Rumäniens mit einer Auflage (1964) von 80.000 Exemplaren. ${ }^{27}$ Der Tod des Diktators war für Leser und Redakteure allerdings weniger das Signal zur offenen Berichterstattung als vielmehr zur Auswanderung, mit der Folge, dass die Redaktion auf rd. 30 Mitarbeiter und die Auflage auf 3.000 Exemplare schrumpfte. Brücken zwischen beiden Kulturen schlägt nicht nur das Deutsche Kulturzentrum, das sich für Begegnungen junger Künstler und die Sprachpflege einsetzt und die mit ihm verbundene örtliche Deutsche Kulturgesellschaft, sondern ebenso ein Architekturbüro der Deutschen Gesellschaft für Internationale Zusammenarbeit (GIZ), das sich nach erfolgreichem Wirken in Hermannstadt (Sibiu) nun um die Sanierung der Stadt Temeswar kümmert. Fügt man dem schießlich die zahlreichen Filialen deutscher und österreichischer Unternehmen, Nichtregierungsorganisationen oder Galerien hinzu, dann findet der Fremde aus Deutschland in Temeswar als Gebender und Nehmender permanent offene Türen und keinen Grund zur Einsamkeit.

\section{Unter Kollegen}

Wenn Herder-Dozenten vortragen, mit Kollegen des Gastlandes eine „Kooperation unter Gleichen ${ }^{\text {“28 }}$ zu praktizieren, so mag dies im Einzelfall wohl stimmen. Wie die heimischen Kollegen übernehmen sie ein volles Lehrdeputat, sind ebenso für Leistungsnachweise, Prüfungen, Beratungen, Sprechstunden, Gutachten, und den regelmäßigen Studienbetrieb verantwortlich, teilen mit den Kollegen sehr enge Dienstzimmer, knappe Material- und Literaturbestände und werden für die Zeit ihres Aufenthalts Steuerbürger des Gastlandes. Der Lehrbetrieb bringt sie zusammen, doch ist der streng geregelt und lässt sehr wenig Raum für Spontaneität. Mit der Zeit schärft sich aber auch der Blick dafür, in der universitären Gleichheit die Ungleichheiten zu entdecken. Sie äußern sich in der abweichenden Verteilung personeller Ressourcen von Abteilung zu Abteilung, von Fach zu Fach oder auch in unterschiedlichen Zugangschancen zu Leitungspositionen. Kollegen und Verwaltungsmitarbeiter begegnen den Gästen zwar freundlich, zugleich aber distanziert; enger, ja familiär wird die Kommunikation nur mit jenen, mit denen man kontinuierlich zu tun hat.

27 Der Tagesspiegel vom 02.05.2008.

28 Krauss, H.-L.: Przepraszamt! Leider kann ich kein Polnisch..., in: Begegnungen, a.a.O., 48-52, 49. 
Die erlebte größtmögliche Distanz ergibt sich aus dem durchaus erkennbaren Wunsch anderer, die West-Universität doch wieder verlassen. Er oder sie teilten dies zunächst indirekt durch vorenthaltene Seminarräume mit, dann direkter mit dem Wandtafelsatz: „Raus, raus, dies ist eine rumänische Universität“. Fand aufgrund verschlossener oder bereits besetzter Räume ein Vorlesung nicht statt, entfiel nach den allgemeinen Regeln damit auch der Entgeltanspruch. Wenngleich solcher Zwist insgesamt nur Episode blieb, sorgte er doch über Wochen für Unruhe, bis Studierende in einer Art von Selbsthilfe einen Zweitschlüssel für die Seminarräume zur Verfügung stellten.

Die sich wiederholenden Raumnöte in den ersten Semestern dokumentierten im Übrigen Gegenwind bereits seitens des zuständigen Dekanats. So sahen sich die Gastdozenten mit der deutschsprachigen Abteilung mitten in einer hochschulpolitischen Kontroverse zwischen Traditionalisten und Reformern. In ihr ging es um die universitätspolitische Vormacht der juristischen und ökonomischen Fächer, die den Rektor stellten, den Akademischen Senat beherrschten und von einer kleineren „Gruppe für die Erneuerung der Universität“ herausgefordert wurden, unter Einschluss des Leiters der deutschsprachigen Abteilung. Unmittelbarer Streitpunkt waren einbehaltene Honorare. Vor allem forderten die Reformer, an der Universität von gewissen Erbschaften aus kommunistischer Zeit abzulassen, analog zur im Jahr 2006 erfolgten offiziellen Verdammung der kommunistischen Diktatur Rumäniens als ein Unrechtsregime. Mit diesen Erbschaften waren nicht nur Personen mit entsprechender Vergangenheit und bezweifelbarer wissenschaftlicher Qualifikation gemeint, sondern auch Verfahren wie der unlautere Erwerb von akademischen Titeln, die Praxis des Plagiierens, die Vorteilsnahme im Amt sowie Begünstigungen bei der Zuteilung von Promotionsrechten und Forschungspotentialen. Auch in diesem Kontext wurde die Möglichkeit zum Notenkauf kritisiert, nach der ,manche Professoren für Barzuwendungen empfänglich sind und im Gegenzug bessere Noten erteilen“29 oder sich überhaupt erst danach einer Prüfung zuwenden. Da sich die Reformer universitätskritisch auch in den Massenmedien äußerten, wurde im Akademischen Senat die fristlose Entlassung des Abteilungsleiters betrieben. Erst großer öffentlicher Zuspruch für die Reformer und nicht zuletzt ein go in der Studierenden ließen den Entlassungsantrag von der Tagesordnung verschwinden. Für die Stu-

29 Wagner, T.: Geld für gute Noten, in: Deutschlandfunk, 1.11.2007, 14.35 Uhr, abgedruckt in Allgemeine Deutsche Zeitung vom 7.11.2007; vgl. auch: Ende der Uni-Krise von Temeswar?, in: Allgemeine Deutsche Zeitung vom 26.11.2007. 
denten war es Mutprobe und Feuertaufe zugleich. Ihre Wortführer stellten sich unter den Leitspruch von Perikles: „Wer an den Dingen der Stadt keinen Anteil nimmt, ist kein stiller, sondern ein schlechter Bürger.“

Dieser Erfolg der Reformer wurde durch zwei Faktoren begünstigt: die starke Resonanz des Konflikts in der Öffentlichkeit und ein demokratisch-liberales universitäres Wahlrecht, das die Besetzung der Hochschulgremien in Wahlen strikt von unten nach oben organisiert. Der Temeswarer Vorgang fand im Übrigen eine Entsprechung in Bukarest, wo Hochschullehrer eine „Koalition für saubere Universitäten“ gründeten. Er war auch ein Erfolg für die maßgeblich vom Philosophen und Soziologen Marga, dem langjährigen Rektor der dreisprachigen Klausenburger Babes-Bolyai Universität, entworfene rumänische Bildungsreform (die er zwischen 1997 und 2000 als Minister verantwortete). Die Reform half, vom quantitativ bewerteten extensiven Unterricht abzurücken hin $\mathrm{zu}$,einer an wissenschaftlichen Leistungen orientierten Ausbildung (...), die sich auf die Erkenntnisse und auf die Gesellschaft auswirkt"“.30 Die strukturellen Probleme der rumänischen Hochschullandschaft - bedeutungslose Forschung, Missbrauch der Bezeichnung Universität, Mangel an Spezialisten, schwache Korrelation zwischen Lehrprogrammen und Arbeitsmarktanforderungen - sollte eine reformierte Universität mit der Einheit von Lehre, Forschung, Lernen und Dienstleistungen auflösen. ${ }^{31}$

Wie die krisenhafte Zuspitzung an der West-Universität demonstrierte, ist der Reformerfolg in starkem Maße von einer veränderten Haltung und Praxis des Hochschulmanagements abhänhig. Die Bedingungen dafür schufen die Reformgesetze, die den Hochschulen bis hinunter zur Abteilungsebene grundlegende Kompetenzen übertrugen. Globalfinanzierung und Studiengebühren versetzten die Universität in Verbindung mit eigenständiger Studentenzulassung, eigener Gehaltsregelung und eigenständigen internationalen Aktivitäten in die Lage, sich dem Typus einer unternehmerischen Hochschule anzunähern. Indem die Hochschulen sich gleichsam neu erfanden, schufen sie die Voraussetzungen, verbindliche internationale Vereinbarungen zu schließen, auch mit der Stiftungsinitiative Johann Gottfried Herder. Die traditionell niedrigen Hochschulgehälter konnten sie allerdings erst im neuen Finanzrahmen nach dem EU-Beitritt deutlich anheben. 


\section{Internationalisierung der Wissenschaft}

Als Joachim Jens Hesse anregte, die Erfahrungen als Herder-Dozent in Osteuropa zu Papier zu bringen, ließ er leichte Zweifel erkennen, ob durch Gastprofessuren eine dauerhafte Wissenschaftskooperation zwischen Deutschland und Osteuropa sich gegen den allgemeinen Westtrend der Internationalisierung der Wissenschaft durchsetzen könne; offensichtlich sei die Kooperationsdichte in der westlichen Welt nach wie vor signifikant höher. Tatsächlich ist der „Westtrend“, ausweislich der verfügbaren Mobilitätsdaten, ungebrochen. So strebten etwa im Jahr 2007 etwa 57 Prozent der international mobilen deutschen Wissenschaftler nach Nordamerika, 27 Prozent nach Westeuropa und ganze 3 Prozent in die Länder Mittel- und Osteuropas. Umgekehrt stammten aber gerade aus diesen Ländern 19 Prozent der ausländischen Forscher in Deutschland, während aus Asien 26 Prozent an, aus Westeuropa 32 Prozent und aus Nordamerika lediglich 12 Prozent der zugereisten Wissenschaftler kamen. ${ }^{32}$ Wie die Daten dokumentieren, kann Deutschland als Wissenschaftsstandort nicht mit der weltweit führenden Wissenschaftsnation, den USA, gleichziehen, doch verfügt das Land als Regionalmacht in europäischer Mittellage über beträchtliche Anziehungskraft, die es in östlicher Richtung stärker zu nutzen gilt.

\section{Konsolidierungsprozesse}

Welche Effekte, besser: Wirkungen, verbinden sich letztlich mit Herder-Gastdozenturen? Subjektiv gesehen waren die acht Semester, über die hier berichtet wird, für die Lehre im Kern des Curriculums erfolgreich, die in Temeswar Studierenden erzielten im Vergleich gute Abschlüsse, konnten sich anschließend in der Konkurrenz um Stipendien, Studienplätze, Projekte und Praktika durchsetzen. Nicht wenige studieren inzwischen in Masterprogrammen an deutschsprachigen Universitäten. Mit Ausnahme der Forschungsaufgabe wurden mithin alle Funktionen, die Parsons den modernen Universitäten zuschrieb, erfüllt: eine akademische Berufsausbildung für Studierende, ihre allgemeine Bildung im Sinne einer nationalen und kulturellen Selbstaufklärung, die Förderung des wissenschaftlichen Nachwuchses sowie Forschungsaktivitäten. ${ }^{33}$ Es spricht mithin einiges dafür, in der Dozentur einen Beitrag zur Konsolidierung, hier des fremdsprachigen Studiengangs Internationale Beziehungen und Europa-Studien, zu 
erkennen. Wissenschaftler wie Studierende verfügen inzwischen über ein solides Instrumentarium internationaler Wissenschaftspraxis.

In der Zwischenzeit wurde der Studiengang vom Bukarester Bildungsministerium akkreditiert und als Fachrichtung in die höchste Stufe der landesweit üblichen Klassifikation eingruppiert. Die meisten der Studierenden erhalten gebührenfreie Plätze, die darüber hinaus erhobenen Gebühren liegen bei 500 Euro pro Platz und Jahr. Die deutschsprachige Abteilung konnte zudem eine ausreichende Zahl von Nachwuchswissenschaftlern, Lektoren und Assistenten anstellen, sodass seit geraumer Zeit wenigstens 80 Prozent der Lehrveranstaltungen durch eigene Kräfte angeboten, Gastdozenturen mithin dauerhaft nicht mehr benötigt werden. Dem Abteilungsleiter wurde im Jahr 2011 zusätzlich das Amt des Direktors der Zentralen Universitätsbibliothek, einer von vier dem Bildungsministerium unterstehenden rumänischen Bibliotheken, übertragen.

\section{Alte und neue Prägungen}

Zwar schreiben sich Semester für Semester zwei bis drei Studierende aus Deutschland oder Österreich in Temeswar ein und stehen damit für ein Mindestmaß an Internationalität des Studiengangs. Als Basis und insbesondere für ein zukunftsbezogenes Profil fehlt dem Ausbildungsgang aber weiterhin eine fachbezogene deutsch-rumänische Universitäts- oder Fakultätspartnerschaft, während über alle Fächer hinweg die stark gestiegene Gesamtzahl deutschrumänischer Kooperationen zu den Positiva der jüngeren Entwicklung zählt.

Tabelle 2: Kooperationsbeziehungen zwischen deutschen und rumänischen Hochschulen und Wissenschaftseinrichtungen 1993-2012 (Anzahl der Kooperationen und Beteiligungen)

\begin{tabular}{c|c|c|c}
\hline Jahr & Kooperationen & Deutschland & Rumänien \\
\hline 1993 & 37 & 28 & 18 \\
\hline 2004 & 176 & 75 & 38 \\
\hline 2012 & 344 & 123 & 49 \\
\hline
\end{tabular}

Quelle: Hochschulrektorenkonferenz/Smolarczyk, R./Referat C4 (Hg.): Mittel- und Osteuropa, Zentralasien, 2012.

Einschränkend ist allerdings hinzuzufügen, dass es sich bei den ausgewiesenen Kooperationen häufig lediglich um zwischenuniversitäre Vereinbarungen im Rahmen des Erasmus-Stipendienprogramms der EU handelt. So weist die WestUniversität 2012 zwar insgesamt 27 Kooperationsbeziehungen aus, von denen aber allein 22 Erasmus-Abreden betreffen und nur fünf allgemeinere Hochschul- 
vereinbarungen sind, die auch nur in zwei Fällen das Themenfeld Forschungszusammenarbeit ansprechen.

Als Folge von Deportation und Aussiedlung der Rumänien-Deutschen sowie als Resultat von Vernichtung und Auswanderung der rumänischen Juden, so Marga im Jahr 2007 anlässlich eines deutsch-rumänischen Seminars, „hat unser Land zwei Riesenverluste erlitten, zwei der größten seiner Geschichte“ “. ${ }^{34}$ Doch bleibe die Hoffnung, die gemeinsame Geschichte auch unter neuen Voraussetzungen nicht zu beenden, sondern fortzuschreiben. Nicht zuletzt dieser historische Kontext ist es, der dem Wirken der entsandten Fremden einen bestimmten Elan (und die entsprechende Motivation) vermittelt. Dabei spielt auch eine Rolle, dass traditionelle kulturelle Orientierungen der rumänischen Bevölkerung aus der Vorkriegszeit offenbar nicht gänzlich verloren gingen, unter denen germanophile Einstellungen in bestimmten Regionen und frankophile Präferenzen in anderen Räumen, vor allem in der Region Bukarest, weit verbreitet waren. Fand dies schon damals seinen Ausdruck in entsprechenden Studienwünschen, so fällt heute erneut auf, dass die Zahlen der rumänischen Studierenden in Deutschland und Frankreich etwa gleich hoch sind. Ein Studium in Deutschland erfolgt erfahrungsgemäß vor allem aus drei Gründen: der Reputation der deutschen Hochschulausbildung, der Lebenssicherheit im Alltag und dem liberalen Arbeitsmarkt für Studierende.

Gemessen an dem, was heute und künftig die Internationalität von Hochschulen ausmachen sollte, sind die Mobilitätsziffern für Studierende und Wissenschaftler allerdings weniger hoch zu bewerten und gelten eher als Selbstverständlichkeit. Nur der Austausch und die gelegentliche Beteiligung an internationalen Forschungs-, Konferenz- oder Publikationsprojekten reichen daher für Deutschlands Position in der globalisierten Welt schon aus Konkurrenzgründen nicht mehr aus. Zumindest konstatiert die „Internationale Strategie der HRK“ (2008 von den Hochschulrektoren verabschiedet), dass die Anschlussfähigkeit an führende internationale Entwicklungen nicht ohne eine stärkere Hinwendung der deutschen Hochschulen zu unternehmerischen Formen der Selbststeuerung, Finanzierung und Profilbildung erreicht werden kann; stärker international und interkulturell ausgerichtete Studiengänge und Forschungsprogramme treten als Desiderate hinzu. ${ }^{35}$ Die ausgreifende Strategie der HRK geht dabei einher mit

34 Rede am 7.10.2007 in Klausenburg, Ms., 2f.

35 Hochschulrektorenkonferenz: Die deutschen Hochschulen internationalisieren! Internationale Strategie der HRK, in: Beiträge zur Hochschulpolitik, 2/2012, Bonn 2012, 7-18, $13 \mathrm{ff}$. 
der „Initiative Außenwissenschaftspolitik“ des Auswärtigen Amtes (ebenfalls aus dem Jahr 2008) sowei weiteren Internationalisierungsansätzen der Ministerien. Außenminister Steinmeiers Grundidee lief darauf hinaus, „Wissenschaftsund Innovationshäuser“ in den Metropolen der Welt zu gründen, die Kooperationen, Infrastrukturen und Netzwerke der einzelnen deutschen Wissenschaftseinrichtungen und -großorganisationen bündeln, das deutsche Angebot im Ausland mithin sichtbarer und leichter nutzbar machen und schließlich die internationalen Aktivitäten deutscher Wissenschaftler befördern sollten. Dem Programm des Auswärtigen Amtes verdanken sich inzwischen Wissenschafts- und Innovationshäuser in New York, Sao Paulo und Neu Delhi sowie ein Wissenschaftszentrum in Kairo; weitere Häusern, u.a. in Tokyo und Moskau, sind geplant. ${ }^{36}$

Gemäß diesen neueren Initiativen verfolgt die akademische Ausbildung künftig das Ziel, ,junge Menschen nicht nur berufsfähig zu machen, sondern für die Wahrnehmung eines Weltbürgertums (global citizenship) zu qualifizieren“. ${ }^{37}$ Das kann nur heißen, mit Christian Bode, dem früheren Generalsekretär des DAAD, die Frage zu stellen, ,welche geistigen und moralischen Orientierungen die künftigen Führungskräfte dieser Welt brauchen, um die Globalisierung friedlich, fair und ertragreich für alle zu gestalten“. ${ }^{38}$ Genau dieser Frage widmen sich Herder-Gastdozenten, allerdings in eher individualistischer Manier und ohne erklärtes Mandat. Der sozial- und geisteswissenschaftliche Herder-Dozent der Zukunft sollte von beiden Seiten, von der gebenden wie der nehmenden, mit einem Mandat versehen werden, daran mitzuwirken, dauerhafte Formen der Zusammenarbeit unter Gleichen, etwa über Universitätspartnerschaften, zu etablieren. Stärker als bisher wäre er dann Akteur in zukunftsbezogenen wissenschaftlichen Kooperationsbeziehungen.

36 Vgl. zum Gesamtkomplex Hesse, J.J.: Die Internationalisierung der Wissenschaftspolitik. Nationale Wissenschaftssysteme im Vergleich, Berlin, 2011.

37 Ebd., 9.

38 Bode, Ch.: Internationalisierung - Status Quo und Perspektiven, in: Borgwardt, A.: Internationalisierung der Hochschulen, hg. v. Friedrich Ebert Stiftung 2012, 7-17, 16. 